\title{
Promoting Health Literacy Is A Necessary Action on the Outskirts Based on the Real Condition There
}

\author{
Majid Khadem-Rezaiyan ${ }^{1}$, Maliheh Dadgarmoghaddam², Ali Sabbagh Gol ${ }^{3}$
}

\footnotetext{
${ }^{1}$ Resident of Community Medicine, Department of Community Medicine and Public Health, Faculty of Medicine, Mashhad University of Medical Sciences, Mashhad, Iran

${ }^{2}$ Specialist in Community Medicine, Assistant Professor, Department of Community Medicine and Public Health, Faculty of Medicine, Mashhad University of Medical sciences, Mashhad, Iran

${ }^{3}$ Resident of Psychiatry, Department of Psychiatry, Faculty of Medicine, Mashhad University of Medical sciences, Mashhad, Iran
}

\section{Type of article: Original}

\begin{abstract}
Introduction: Health literacy refers to the degree to which individuals have the capacity to obtain, process, and understand the basic health information needed to make appropriate health decisions. The aim of this study was to determine the health literacy level in regions on the outskirts of Mashhad, Iran.

Methods: This cross-sectional study was undertaken in December 2014 on residents in the outskirts of Mashhad. We used a multistage sampling method, and data were collected via a questionnaire, conducted by trained interviewers. The data were analyzed using the t-test, the Mann-Whitney test, and the chi-squared test.

Results: We had 502 participants in this study. The mean age was $35.0 \pm 11.06$ years. Sixty percent of participants (301) were female, and $86.3 \%$ (425) had high school diplomas or lower educational levels; the rest had higher educational levels. Television/radio and the Internet were the most and least frequently accessed and used media, respectively. There were significant correlations between the level of knowledge and response to questions in specific domains, such as weight, fasting blood sugar, and blood pressure $(\mathrm{p}<0.001)$. The participants had no difficulty reading booklets (63\%), physicians' prescriptions (59\%), medical forms (56\%), and guidance boards in hospitals (71\%). People had no problems understanding most health/disease materials obtained via various routes (37\%), but the health/disease materials published on the Internet/electronic resources $(37 \%)$ and textbooks $(64 \%)$ were difficult for them to understand.

Conclusion: Health literacy is a complicated construct, and more comprehensive studies are needed to develop health-related information that can be understood by more of the general public.

Keywords: health literacy, outskirts, health-related information
\end{abstract}

\section{Introduction}

Health literacy is one of the most important priorities in promoting the quality of health services (1). Traditionally, health literacy includes the ability to perceive and use the information that is given to patients by health workers. Its functions include the ability to read and understand doctors' prescriptions and instructions or pamphlets. George Bernard Shaw said, "The main problem with communication is the assumption that it has occurred," a universal truth that transcends reading ability (2). Healthy People 2010 (that was designed to measure progress over time, and, most importantly, to clearly lays out a series of objectives to bring better health to all people in the world) uses the following definition for this issue: "The degree to which individuals have the capacity to obtain, process, and understand basic health information and services needed to make appropriate health decisions" (3). It has been shown that this ability is not necessarily related to general reading ability or years of education $(4,5)$. Studies conducted in the United States have shown that the prevalence of inadequate health literacy was $48 \%$. Not surprisingly, only $11 \%$ of adults had adequate health literacy (6). However, a meta-analysis of 85 U.S studies

\section{Corresponding author:}

Assistant Professor Dr. Maliheh Dadgarmoghaddam, Department of Community Medicine, Faculty of Medicine , Mashhad University of Medical sciences, Mashhad, Iran. Tel: +98.5138002398, Email: Dadgarmm@mums.ac.ir Received: September 05, 2015, Accepted: October 27, 2015, Published: January 2016 iThenticate screening: October 27, 2015, English editing: December 20, 2015, Quality control: January 05, 2016 (C) 2015 The Authors. This is an open access article under the terms of the Creative Commons Attribution-NonCommercialNoDerivs License, which permits use and distribution in any medium, provided the original work is properly cited, the use is non-commercial and no modifications or adaptations are made. 
concluded that the prevalence of inadequate and borderline health literacy were $25 \%$ and $20 \%$, respectively (7). Despite great differences in socio-demographic status, the situation in Iran is somewhat similar. A comprehensive study conducted in five provinces in Iran showed that health literacy was adequate, marginal, and inadequate in $28.1,15.3$, and $56.6 \%$ of the participants, respectively. There also were significant associations between inadequate health literacy and old age, low education, and economic status (8). Correlations between inadequate health literacy and poor communication with physicians, less participation in making decision about treatment, failure to follow doctor's orders, improper use of medications, and poor self-care in patients have been noted. More importantly, from a public health perspective, people with inadequate health literacy receive less preventive services and more frequently use emergency departments and hospital services (9). As a consequence, lower health literacy can lead to undesirable social, economic, and health outcomes, such as increasing health and treatment expenses, morbidity, mortality and side effects of diseases $(10,11)$. To date, the few studies that have been conducted in Iran have generally estimated the prevalence of health literacy in different populations in urban regions $(1,8,11-13)$, while no research has been performed that focused on regions in the suburban areas. Low health literacy is not a just a problem for patients; it is a great challenge for the health system as a whole in each country. By considering the importance of the possible results of health literacy on healthcare workers' ability to communicate with patients, as well as the fact that they often are not aware of the health literacy level of their audience. The aim of this study was to assess health literacy levels in regions on the outskirts of Mashhad (the second most populous city in Iran and capital of Razavi Khorasan Province), which is located in northeast Iran).

\section{Material and Methods}

\subsection{Research design and setting}

This cross-sectional study was conducted in December 2014 in Mashhad, Iran.

\subsection{Sample size and sampling method}

A sample size of at least 400 people was calculated as being required to show the prevalence of inadequate health literacy $\left(53 \%\right.$ ) (Using this formula: $\mathrm{n}=\mathrm{Z}^{2} \mathrm{pq} / \mathrm{d}^{2}$ ), with a study power of $80 \%$ and level of significance at $\alpha=0.05$. Ultimately 500 people were surveyed to cover possible dropouts.

Multi-stage random sampling was conducted so that a proportional sample size was dedicated to each region on the outskirts of Mashhad. (According to health care settings, there are five regions in this city.) In each region, five districts were selected using a random number table. In each district, the interviewer faced toward Qibla and turned clockwise until he/she encountered the first alley. Then, he/she started to recruit participants in the study based on inclusion and exclusion criteria, until the size of that cluster was fulfilled.

\subsection{Inclusion criteria}

The inclusion criteria were willingness to participate in the study and age between 18-65 years.

\subsection{Measurement tool}

The questionnaire was designed by the Educational and Health Promotion Office of the Health Ministry of Iran, and its reliability and validity were shown previously (8). Although the validity and reliability of this questionnaire were confirmed in previous study (14), we calculated Cronbach's alpha for this population and found it to be 0.93 . This questionnaire included questions about basic demographic information, along with questions for assessing different dimensions of health literacy (Reading (5 items), Understanding (14 items), Access (6 items), Appraisal (8 items), Decision (12 items).

\subsection{Data collection}

Prior to implementation of the study, five teams (a man and a woman in each team to ensure the reliability of answers and respect for cultural values) who worked as healthcare volunteers (with two years' experience as a health care worker and a high school diploma as a minimum degree), were trained to fill out the questionnaire and communication skills, and probable difficulties were discussed. When the interviewer reached each home, the main objectives of the study were explained to the participants, and their consent to participate in the study was obtained. Then, the questionnaire was completed by researcher after interviewing the participants.

\subsection{Research ethics}

The Ethics Committee of Mashhad University of Medical Sciences approved this study (930548). Oral informed consent was obtained from the participants. The main ethical considerations in this study were: 
1) We were honest with participants about the length of interview and the purpose of the study.

2) Participation in this study was voluntary.

3) Any of the particiants could exit the study at any time during the interview.

4) The information will be published without personally-identifiable information (PII).

5) Each gender participated in the interview teams to ensure appropriate consideration of cultural values.

\subsection{Statistical analysis}

The data were analyzed statistically by the Statistical Program for Social Sciences (SPSS), version 11.5 (SPSS Inc., Chigaco, Illinois, United States) using the t-test, the Mann-Whitney test, and the chi-squared test (goodness of fit) to examine differences and correlations between responses. A level of $\alpha<0.05$ was considered to be statistically significant.

\section{Results}

We interviewed 502 participants, of whom $60 \%$ (301 participants) were females. The mean age $( \pm \mathrm{SD})$ of the study population was $35.0 \pm 11.06$. Among the participants, $425(86.3 \%)$ had a high school diploma or a lower educational level; the rest had a higher educational level. Participants' responses about access to and usage of health information are shown in Figure 1. As the chart shows, the most and least frequent methods both for accessing information and using it were radio/television and the Internet, respectively. The questionnaire included five questions regarding the reading difficulty of educational packages about health. The participants had no difficulties reading booklets (63\%), physicians' prescriptions (59\%), medical forms (56\%) and guidance boards in hospitals (71\%) (Table 1).

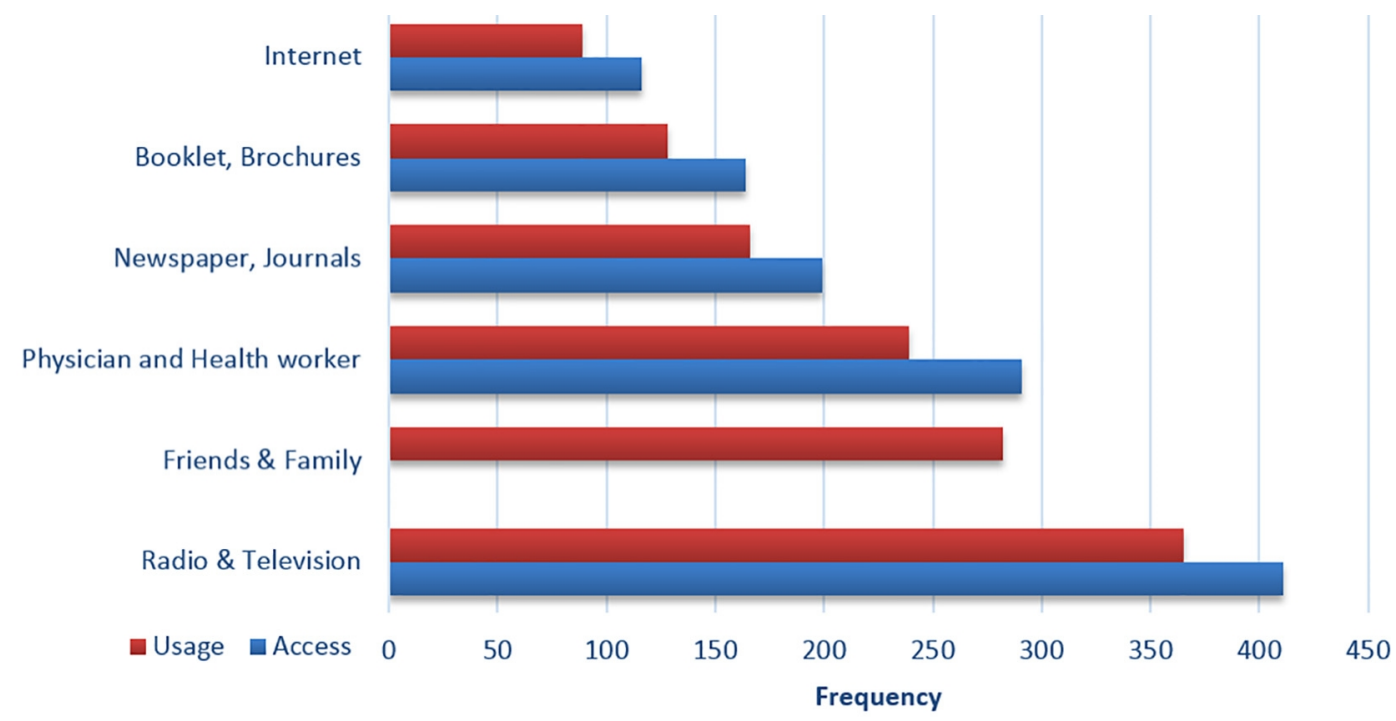

Figure1. Different methods used by participants for accessing and using health information

Table 1. Reading difficulty domain

\begin{tabular}{|l|l|l|l|l|}
\hline Questions & Very Easy & Easy & Not Easy, Not Hard & Very Hard \\
\cline { 2 - 5 } & $\mathrm{n}(\%)$ & $\mathrm{n}(\%)$ & $\mathrm{n}(\%)$ & $\mathrm{n}(\%)$ \\
\hline $\begin{array}{l}\text { Educational or commercial Booklet } \\
\text { brochures about health }\end{array}$ & $139(28)$ & $173(34.8)$ & $122(24.5)$ & $63(12.7)$ \\
\hline $\begin{array}{l}\text { Written prescriptions of physicians or } \\
\text { health work force about health or disease }\end{array}$ & $131(26.3)$ & $166(33.3)$ & $125(25.1)$ & $77(15.4)$ \\
\hline $\begin{array}{l}\text { Subjects in Medical or dentistry forms in } \\
\text { hospitals or healthcare centers }\end{array}$ & $135(27.3)$ & $139(28.1)$ & $139(28.1)$ & $82(16.6)$ \\
\hline $\begin{array}{l}\text { Guidance boards in hospitals, clinics and } \\
\text { healthcare centers }\end{array}$ & $169(33.8)$ & $183(36.6)$ & $97(19.4)$ & $51(10.2)$ \\
\hline $\begin{array}{l}\text { Guidance protocols before the laboratory } \\
\text { tests, sonography or radiological } \\
\text { interventions }\end{array}$ & $157(31.6)$ & $147(29.6)$ & $113(22.7)$ & $80(16.1)$ \\
\hline
\end{tabular}


There were 14 questions regarding the understanding and interpretation of health issues. People had no problems understanding most health/disease materials obtained via various routes $(37 \%)$; however, health/disease materials published on the Internet/electronic resources (37\%) and textbooks (64\%) were difficult for them to understand (Table 2). Perceived knowledge about weight was significantly correlated with the participant's response to questions about this issue $(\mathrm{p}<0.001)$ (Figure 2). Women were more likely than men to answer weight-related questions correctly $(\mathrm{p}=0.008)$. Perceived knowledge about fasting blood sugar $(\mathrm{FBS})$ was significantly correlated with responses to questions about this issue $(\mathrm{p}<0.001)$ (Figure 3$)$. There was no significant difference between the responses of the two genders $(\mathrm{p}=0.3)$. Perceived knowledge about blood pressure $(\mathrm{BP})$ was significantly correlated with answers to questions about this issue $(\mathrm{p}<0.001)$ (Figure 4$)$. Women had more correct answers than men $(\mathrm{p}=$ 0.009).

Table 2. Understanding and interpretation domain

\begin{tabular}{|c|c|c|c|c|c|}
\hline \multicolumn{2}{|l|}{ Questions } & \multirow{2}{*}{$\begin{array}{l}\text { High } \\
\mathrm{n}(\%)\end{array}$} & \multirow{2}{*}{$\begin{array}{l}\text { Moderate } \\
\mathrm{n}(\%)\end{array}$} & \multirow{2}{*}{$\begin{array}{l}\text { Low/No } \\
\mathrm{n}(\%)\end{array}$} & \multirow{2}{*}{$\begin{array}{l}\text { Not } \\
\text { Applicable } \\
\mathrm{n}(\%)\end{array}$} \\
\hline & & & & & \\
\hline \multirow[t]{8}{*}{ Understanding } & $\begin{array}{l}\text { Guidance boards in hospitals, clinics and } \\
\text { healthcare centers }\end{array}$ & $157(31.6)$ & $147(29.6)$ & $113(22.7)$ & $80(16.1)$ \\
\hline & $\begin{array}{l}\text { Guidance protocols before the laboratory } \\
\text { tests, sonography or radiological } \\
\text { interventions }\end{array}$ & $176(35.4)$ & $188(37.8)$ & $101(20.3)$ & $32(6.4)$ \\
\hline & Expert opinions in Radio/TV & $225(45.4)$ & $199(40.1)$ & $53(10.7)$ & $19(3.8)$ \\
\hline & $\begin{array}{l}\text { Health/disease materials in Internet and } \\
\text { electronic resources }\end{array}$ & $83(16.9)$ & $97(19.8)$ & $82(16.7)$ & $229(46.6)$ \\
\hline & Health/disease materials in textbooks & $79(16)$ & $143(28.9)$ & $171(34.6)$ & $101(20.4)$ \\
\hline & $\begin{array}{l}\text { Health/disease materials in the } \\
\text { newspaper, booklet and brochures }\end{array}$ & $168(34.3)$ & $170(34.7)$ & $99(20.2)$ & $53(10.8)$ \\
\hline & $\begin{array}{l}\text { Drug prescription orders that have been } \\
\text { written on drugs }\end{array}$ & $275(55.4)$ & $145(29.2)$ & $51(10.3)$ & $25(5)$ \\
\hline & $\begin{array}{l}\text { Physician and healthcare worker } \\
\text { consultations }\end{array}$ & $261(52.6)$ & $172(34.7)$ & $49(9.9)$ & $14(2.8)$ \\
\hline \multirow[t]{6}{*}{ Interpretation } & Information from radio/TV & $233(46.5)$ & $199(39.7)$ & $55(11)$ & $14(2.8)$ \\
\hline & Information from Internet & $91(18.4)$ & $92(18.6)$ & $76(15.4)$ & $236(47.7)$ \\
\hline & Recommendations from physicians & $213(42.6)$ & $199(39.8)$ & $67(13.4)$ & $21(4.2)$ \\
\hline & $\begin{array}{l}\text { Information from educational or } \\
\text { commercial booklets or brochures }\end{array}$ & $165(33.3)$ & $187(37.7)$ & $100(20.2)$ & $44(8.9)$ \\
\hline & Information from newspaper, journal & $169(34.1)$ & $178(36)$ & $96(19.4)$ & $52(10.5)$ \\
\hline & $\begin{array}{l}\text { Recommendations from friends or } \\
\text { relatives }\end{array}$ & $252(50.5)$ & $174(34.9)$ & $53(10.6)$ & $20(4)$ \\
\hline
\end{tabular}

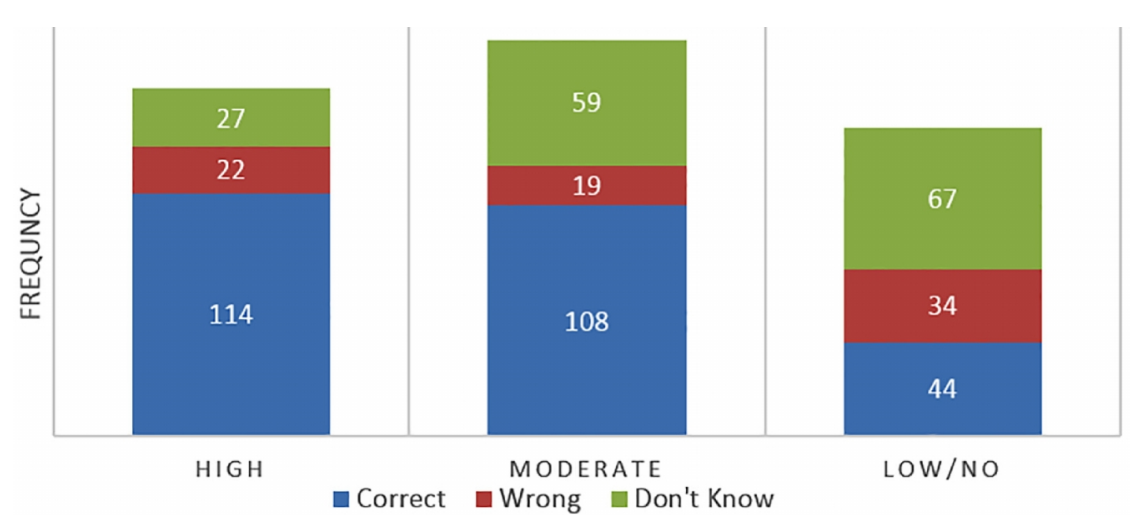

Figure 2. Frequency of different answers about weight based on perceived knowledge of participants 


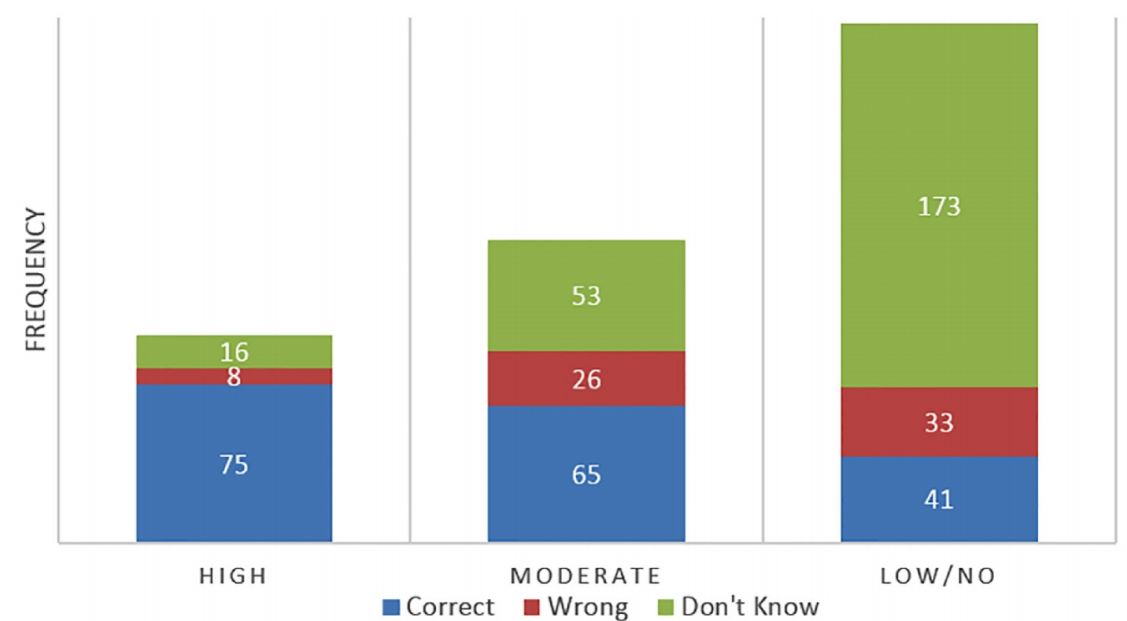

Figure 3. Frequency of different answers about FBS based on perceived knowledge of participants

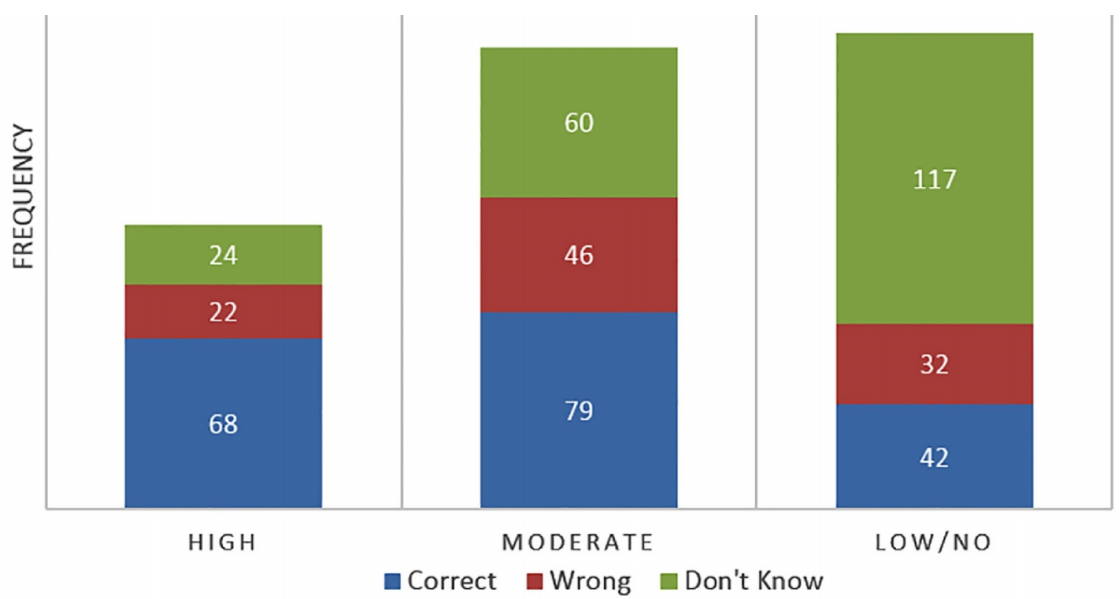

Figure 4. Frequency of different answers about blood pressure based on perceived knowledge of participants

\section{Discussion}

In the past decade, deficiencies in health literacy were ringing warning bells for those working in the healthcare system. Various researchers have suggested that clinicians should adjust how they communicate based on the actual level of patients' health literacy (e.g., simple language, low speed, and including family members in discussions) (2, 15). Low communication of health literacy was shown previously in Mashhad (10); but this study showed that healthcare professionals were actually the second choice for accessing and using health information in the outskirts regions. This study showed that the more accessible the media, the more it will be used to gain information on health. As expected, radio/television was the most popular route for gaining information in this study area, while, as a developing country, the Internet was the least commonly-used medium for this purpose. A positive association can be seen between accessibility and usage of a specific method. So, trying to shift people to use the Internet, for example, is not possible until acceptably high-speed Internet connections are available all over the country. The participants had no difficulties in reading booklets, physician prescriptions, medical forms, and guidance boards in hospitals. Since this study was done in the outskirts regions, the results of the study may be related to the war against illiteracy that began 30 years ago when the "Literacy Movement" was established. The latest reports from the Ministry of Education show that there are still nine million illiterate people in Iran (16). People had no problem in understanding most health/disease materials available via various routes, indicating that, after reading the material, they had a high-to-moderate level of understanding about the topic of the materials. However, health/disease materials available on the Internet or in electronic resources and textbooks were difficult for them to understand. As mentioned above, Internet access and usage were the least commonly-used way of accessing health information, and this could account for the difficulties found in processing this information. The National Work Group on Literacy and Health in the United States has suggested that health material should be written at a level suitable for children in the fifth grade, which is still difficult for $25 \%$ of the community (17). When it came to interpretation of information, 
the findings were congruent with the domain relating to access and usage. Information obtained from radio/television was the easiest for the respondents to interpret. This could explain why these two media are the most popular routes for obtaining health/disease information for lay people. Three questions were asked to evaluate the participants' perceived health knowledge. The most correct answers were given for questions about weight, with FBS and BP in second and third place, respectively. Approximately half of the study sample had little or no information on FBS; however, some of them answered the question correctly. As there was a significant relation between perceived knowledge and correct answers to questions, this can be taken to be a valid marker for the validation of their perceived knowledge. Women gave more correct answers to questions concerning weight and blood pressure, which was incongruent with previous studies in which females had lower health literacy (4, 8, 10). However, our findings were similar to the findings of a nationwide study conducted in Britain (18). This could be due to the nature of our target population (i.e., people in outskirt regions), where there is no significant difference between the educational levels of men and women. Using a questionnaire as a data collection tool has its own limitations. For example, we asked people to state their perceptions of reading, understanding, and interpreting health information, but a test may have been more accurate. In some cases, it was possible to validate their answers using a true/false question, but this was not applicable to all health literacy domains. Since the questionnaire was completed as an interview, we had a response rate of almost $100 \%$. The findings of this study can be generalized to all outskirt regions of Mashhad based on our sampling method. This study has filled a key gap in our knowledge by surveying the population in the outskirts of Mashhad, since nearly all previous projects were performed in urban healthcare centers. However, health literacy is a complicated construct, and more comprehensive studies are needed to develop health-related information that more of the general public can understand.

\section{Conclusions}

This study showed that health literacy was not good in the outskirts, but, as expected, radio/television was the most popular route for gaining information in this study area. So, the health policy makers must consider that the best media for health education and improving health literacy in the outskirts are public media, which impact the vast majority of lay people.

\section{Acknowledgments:}

This project was conducted under the supervision of the Vice Chancellor for Research at Mashhad University of Medical Sciences. Special thanks to the health care volunteers who assisted us in the data collection stage.

\section{Conflict of Interest:}

There is no conflict of interest to be declared.

\section{Authors' contributions:}

All authors contributed to this project and article equally. All authors read and approved the final manuscript.

\section{References}

1) Kohan S, Ghasemi P, Dodangeh M. Associations between maternal health literacy and prenatal care and pregnancy outcome. Iran J Nurs Midwifery Res 2007; 11 (3): 33-44.

2) David W Baker. The Meaning and the Measure of Health Literacy. J Gen Intern Med. 2006; $21(8): 878-83$. doi: 10.1111/j.1525-1497.2006.00540.x, PMCID: PMC1831571.

3) U.S. Department of Health and Human Services. Healthy People 2010: Understanding and Improving Health. 2nd ed. Washington, DC: U.S. Government Printing Office, November, 2000.

4) Paasche-Orlow MK, Riekert KA, Bilderback A, Chanmugam A, Hill P, Rand CS, et al. Tailored education may reducehealthliteracy disparities in asthma self-management. Am J Resp Crit Care. 2005; 172 (8): 980 6. doi: 10.1164/rccm.200409-12910C, PMID: 16081544, PMCID: PMC2718412.

5) Norman CD, Skinner HA. eHealth literacy:essential skills for consumer health in anetworked world. J Med Internet Res. 2006; 8(2). doi: 10.2196/jmir.8.2.e9, PMCID: PMC1550701.

6) Yin HS, Johnson M, Mendelsohn AL,Abrams MA, Sanders LM, Dreyer BP. The health literacy of parents in the United States: anationally representative study. Pediatrics. 2009;124:289-98. doi: 10.1542/peds.20091162E, PMID: 19861483.

7) Paasche-Orlow MK, Parker RM, Gazmararian JA, Nielsen-Bohlman LT, Rudd RR. The prevalence of Limited health literacy. J Gen Intern Med. 2005; 20(2): 175-84. doi: 10.1111/j.1525-1497.2005.40245.x, PMCID: PMC1490053. 
8) Tehrani Banihashemi SA, Amirkhani MA,Haghdoost AA, Alavian SM, Asgharifard H, Baradarn H, et al. Health literacy and the affecting factors: a study in five provinces of Iran. Journal of Medical Education Development Center. 2007; 4 (1): 1-9.

9) Peerson A, Saunders M. Health literacy revisited: what do we mean and why does it matter? Health Promot Int. 2009; 24 (3): 285-96. doi: 10.1093/heapro/dap014

10) Peyman N, Behzad F, Taghipour A, Esmaily H. Evaluation of communication between healthcare workers and patients with chronic diseases according to their levels of health literacy. J Research Health. 2014; 4(1): 599-607.

11) Ghanbari S, Majlessi F, Ghaffari M, Majdabadi M M. Evaluation of health literacy of pregnant women in urban health centers of Shahid Beheshti Medical University. Daneshvar Med. 2012; 19(97): 1-12.

12) Reisi M, Mostafavi F, Hasanzadeh A,Sharifirad GR. The Relationship between Health Literacy, Health status and Healthy behaviors among Elderly in Isfahan. J Health Syst Res. 2011; 7 (4): 469-80.

13) Javadzade H, Sharifirad Gh, Reisi M, Tavassoli E, Rajati F. Health Literacy among Adults of Isfahan, Iran. J Health Syst Res. 2013; 9(5): 540-9.

14) Montazeri A, Tavousi M, Rakhshani F, Azin SA, Jahangiri K, Ebadi M, et al. Health Literacy for Iranian Adults (HELIA): develoment and psychometric properties. Payesh. 2014; 13: 589-600

15) Parker R. Health literacy: a challenge for American patients and their health care providers. Health promotion International. 2000; 15(4): 277-83, doi: 10.1093/heapro/15.4.277

16) Comprehensive information websitebase of Ministry of Education of Islamic Republic of Iran. Available from: http://www.medu.ir/Portal/Home. 2014.

17) National Work Group on Literacy and Health. Communicating with patients who have limited literacy skills. Report of the National Work Group Literacy and Health. J Fam Pract. 1998, 46(2): 168-76, PMID: 9487325

18) Von Wagner C, Knight K, Steptoe A, Wardle J. Functional health literacy and health-promoting behaviour in a national sample of British adults. J Epidemiol Community Health. 2007; 61(12): 1086-90. doi: 10.1136/jech.2006.053967, PMID: 18000132, PMCID: PMC2465677. 